\title{
New parametrization of the neutrino mixing matrix for neutrino oscillations
}

\author{
Virendra Gupta \\ Departmento de Fisica Aplicada, CINVESTAV-Unidad Merida, A.P. 73 Cordemex 97310 Merida, Yucatan, Mexico \\ Xiao-Gang He \\ Department of Physics, National Taiwan University, Taipei, Taiwan 10764, Republic of China
}

(Received 27 June 2001; published 8 November 2001)

\begin{abstract}
In this paper we study three active neutrino oscillations, favored by recent data from SuperK and SNO, using a new parametrization of the lepton mixing matrix $V$ constructed from a linear combination of the unit matrix $I$, and a Hermitian unitary matrix $U$, that is, $V=\cos \theta I+i \sin \theta U$. There are only three real parameters in $V$ including the parameter $\theta$. It is interesting to find that experimental data on atmospheric neutrino dictates the angle $\theta$ to be $\pi / 4$ such that the $\nu_{\mu}$ and $\nu_{\tau}$ mixing is maximal. The solar neutrino problem is solved via the Mikheyev-Smirnov-Wolfenstein effect with a small mixing angle, with $U$ depending on one small parameter $\epsilon$. The resulting mixing matrix with just two parameters $(\theta$ and $\epsilon)$ predicts that the oscillating probabilities for $\nu_{e} \rightarrow \nu_{\mu}$ and $\nu_{e} \rightarrow \nu_{\tau}$ be equal and of the order of $2 \epsilon^{2}=(0.25-2.5) \times 10^{-3}$. The measurement of $C P$ asymmetries at the proposed neutrino factories would also provide a test of our parametrization.
\end{abstract}

DOI: 10.1103/PhysRevD.64.117301

PACS number(s): 14.60.Pq, 12.15.Ff

Flavor mixing in both quark and lepton sectors is one of the mysteries of particle physics. There are abundant experimental data that show that different quark generations mix and so do lepton generations. However, theoretical understanding of the mixing is very poor. The minimal standard model (SM) can accommodate quark mixing through the Cabibbo-Kobayashi-Maskawa mixing matrix [1]. For mixing in the lepton sector, one has to extend the minimal SM, either by introducing a new scalar particle to provide Majorana masses for neutrinos and therefore mixing, or by introducing right-handed neutrinos to provide neutrinos with Dirac mass, or a combination of these two. The mixing phenomena in the lepton sector are most prominent in neutrino oscillations and can be described by the Maki-Makagawa-Sakata mixing matrix [2]. These proposals can accommodate mixing phenomena and can be made consistent with experimental data, but there is no understanding why the matrix elements in relevent mixing matrix have the determined values.

In this paper assuming three generations of neutrinos, we consider another way of looking at the mixing matrix problem by dividing the mixing matrix into two pieces. One of them is proportional to the unit matrix $I$ which does not cause any mixing, and the other is proportional to a Hermitian unitary matrix $U$ which is responsible for flavor mixing. The mixing matrix $V$ is thus parametrized as $V=\cos \theta I$ $+i \sin \theta U$. This way of looking at the problem has the advantage that the parameter $\theta$ determines the relative weight of the diagonal elements and off diagonal elements in a nontrivial manner. Mathematically, $U$ depends on four real parameters, but due to the rephasing invariance of $V$, there are actually just two real independent parameters in $U$. A similar proposal has been made for the quark mixing matrix which is compatible with experimental data within allowed errors [3]. Here we will concentrate on the lepton sector to analyze mixing phenomena in neutrino oscillations. Neutrino oscillations can provide natural explanations to the atmospheric and solar neutrino data. Recent data from SuperK [4] and SNO [5] favor mixing of active neutrinos. We therefore assume three active neutrino mixing in our analysis. We note that the liquid scintillation neutrino detector (LSND) [6] data cannot be accommodated in this analysis. The required parameter space to explain the atmospheric and solar neutrino data can provide stringent constraints on the parameters in the mixing matrix. Using atmospheric neutrino data, we find that the linear combination angle $\theta$ is determined to be $\pi / 4$, indicating that the diagonal and off diagonal parts are equally important. The solar neutrino problem can be solved by the small mixing angle (SMA) solution with MikheyevSmirnov-Wolfenstein (MSW) effect. This ansatz also gives interesting predictions for long base line neutrino experiments and $C P$ violation in neutrino oscillations.

We now provide a detailed analysis of the parametrization proposed here. Unitarity of $V$ requires

$$
\begin{aligned}
& V V^{\dagger}=I=\cos ^{2} \theta I+\sin ^{2} \theta U U^{\dagger}+i \sin \theta \cos \theta\left(U-U^{\dagger}\right), \\
& V^{\dagger} V=I=\cos ^{2} \theta I+\sin ^{2} \theta U^{\dagger} U+i \sin \theta \cos \theta\left(U-U^{\dagger}\right) .
\end{aligned}
$$

In general $V$ and $U$ are nontrivially related. However, if $U$ is independent of the linear combination angle $\theta$, then $U$ has to be unitary and Hermitian. The $V$ so obtained is more restrictive than a general one.

The matrix $U$ can be parametrized as

$$
U=\left(\begin{array}{ccc}
-1+2|c|^{2} & -2 b^{*} c & -2 a^{*} c^{*} \\
-2 b c^{*} & -1+2|b|^{2} & -2 a b^{*} \\
-2 a c & -2 a^{*} b & -1+2|a|^{2}
\end{array}\right)
$$

where the magnitudes and the phases of $a, b$, and $c$ satisfy the constraints $|a|^{2}+|b|^{2}+|c|^{2}=1$ and $\phi_{a}-\phi_{b}+\phi_{c}=\pi / 2$, respectively. $U$ depends, in general, on two independent moduli and two independent phases as it stands. However the mixing matrix $V$ is rephasing invariant, so the two phases in $U$ can be absorbed into lepton phases. $V$ depends on only 3 real parameters, $\theta$ and the two moduli in $U$. 
To determine the parameters in the mixing matrix $V$, we first consider data for atmospheric neutrino from SuperK experiments [4]. To fit the data the muon neutrino $\nu_{\mu}$ has to oscillate into another type of neutrino $\nu_{x}$ with mixing angle $\theta_{2 x}$ such that $\sin ^{2} 2 \theta_{2 x}>0.88$, which leads to $\theta_{2 x} \approx \pi / 4$ and also the mass squared difference $\left|\Delta m_{2 x}^{2}\right|=\left|m_{2}^{2}-m_{x}^{2}\right|$ in the range $(1.5-5) \times 10^{-3} \mathrm{eV}^{2}$. Here the numbers 1,2 , and 3 correspond to $\nu_{e}, \nu_{\mu}$, and $\nu_{\tau}$, respectively. For three generations of neutrinos, $\nu_{\mu}$ can only oscillate into a tauon neutrino $\nu_{\tau}$ to fit the data. Assuming that electron neutrino $\nu_{e}$ does not have significant mixing with muon and tauon neutrinos, then $\left|V_{11}\right|$ is fixed to be 1 , while $\left|V_{22}\right|,\left|V_{23}\right|,\left|V_{32}\right|$, and $\left|V_{33}\right|$ are fixed to be $1 / \sqrt{2}$. This leads to the following unique solution for $\theta$ and $U$ :

$$
\begin{aligned}
& \theta=\frac{\pi}{4}, \quad a=\frac{1}{\sqrt{2}}, \quad b=\frac{1}{\sqrt{2}}, \quad c=0, \\
& U=\left(\begin{array}{ccc}
-1 & 0 & 0 \\
0 & 0 & -1 \\
0 & -1 & 0
\end{array}\right) .
\end{aligned}
$$

The mass squared difference $\left|\Delta m_{23}^{2}\right|$ is constrained to be in the range $(1.5-5) \times 10^{-3} \mathrm{eV}^{2}$ at $90 \%$ C.L. The mixing angle $\theta=\pi / 4$ is a reflection of the fact that the mixing of the $\nu_{\mu}$ and $\nu_{\tau}$ is maximal. It is interesting that this value of $\theta$ also implies that the purely diagonal and the nondiagonal pieces have equal weight, i.e., $V=(I+i U) / \sqrt{2}$.

The mixing matrix obtained above cannot produce oscillations of electron neutrino into other neutrinos and therefore cannot provide a solution to the solar neutrino problem. If the solar neutrino problem is due to neutrino oscillation like the solution to the atmospheric neutrino problem, then the electron neutrino has to mix with other neutrinos such that it can oscillate into them to cause the deficit seen in the measurements on earth. One has to modify the above mixing matrix such that solutions for the solar neutrino problem can be obtained.

To this end we make a small perturbation to the mixing matrix by introducing a nonzero but small value for $c$ such that $V_{12,23}$ become nonzero to allow $\nu_{e}$ to oscillate into $\nu_{\mu}$ and/or $\nu_{\tau}$. In fact, in our parametrization, a nonzero value for $c$ implies that both $V_{12}$ and $V_{13}$ are nonzero. Whether the solar neutrino problem is solved by oscillation of $\nu_{e}$ into $\nu_{\mu}$ or $\nu_{\tau}$ depends on the mass squared differences of the neutrino masses that will be discussed later. Denote the perturbations on $a, b$, and $c$ as $a=\left(1+\epsilon_{a}\right) / \sqrt{2}, b=\left(1+\epsilon_{b}\right) / \sqrt{2}$, and $c=i \epsilon$. If $\epsilon$ is a real number, $\epsilon_{a, b}$ are also required to be real from the constraint on the phases. A simple choice, satisfying all constraints, is to take $c=i \epsilon$ with $a$ $=\sqrt{1-2 \epsilon^{2}} / \sqrt{2}$ and $b=1 \sqrt{2}$. This gives

$$
V=\frac{1}{\sqrt{2}}\left(\begin{array}{ccc}
1-i\left(1-2 \epsilon^{2}\right) & \sqrt{2} \epsilon & -\sqrt{2} \epsilon \sqrt{1-2 \epsilon^{2}} \\
-\sqrt{2} \epsilon & 1 & -i \sqrt{1-2 \epsilon^{2}} \\
\sqrt{2} \epsilon \sqrt{1-2 \epsilon^{2}} & -i \sqrt{1-2 \epsilon^{2}} & 1-i 2 \epsilon^{2}
\end{array}\right) .
$$

There are other choices for $\epsilon_{a, b}$, which can also satisfy $|a|^{2}+|b|^{2}+|c|^{2}=1$, such as the general case $a$ $=\sqrt{1-2(1+\alpha) \epsilon^{2}} / \sqrt{2}$ and $b=\sqrt{1+2 \alpha \epsilon^{2}} / \sqrt{2}$, with $\alpha$ a number of order one so that $\epsilon_{a, b}$ are small. However, to the leading order in $\epsilon^{2}$, the general case gives the same results for neutrino oscillations. Without loss of generality, we will carry out the rest of the analysis using $V$ given above.

There are four types of solutions to the solar neutrino problem [7]. Three of them are based on the MSW mechanism [8]. They are the large mixing angle (LMA), the long wavelength large mixing angle, and the small mixing angle (SMA) solutions. There is one solution that does not depend on the MSW effect, the "just so" vacuum oscillation solution. Recent data from SuperK favors the LMA solution [7]. However, at present it is premature to rule out the other three solutions. Among the four solutions, our parametrization can only accommodate the SMA solution. Fitting data, we determine the parameter $\epsilon^{2}$ and the mass squared difference of $\nu_{e}$ and the neutrino oscillated into $\nu_{x}$ to be

$$
\begin{aligned}
\epsilon^{2} & =(0.125-1.25) \times 10^{-3}, \\
\left|\Delta m_{1 x}^{2}\right| & =(0.35-1) \times 10^{-5} \mathrm{eV}^{2} .
\end{aligned}
$$

Because the mixing matrix elements $V_{12}$ and $V_{13}$ to the leading order in $\epsilon$ are equal, present constraints on various neutrino oscillation data can allow the neutrino $\nu_{x}$ to be $\nu_{\tau}$ or $\nu_{\mu}$ depending on the pattern of the neutrino masses. There are two scenarios: (a) if $\nu_{e}$ oscillates into $\nu_{\tau}$, the neutrino mass hierarchy will be $m_{\nu_{\mu}}>m_{\nu_{\tau}}>m_{\nu_{e}}$, and (b) if $\nu_{e}$ oscillates into $\nu_{\mu}$, then $m_{\nu_{\tau}}>m_{\nu_{\mu}}>m_{\nu_{e}}$.

The mass hierarchy patterns for cases (a) and (b) have different implications in general for long base line experiments. The probability for an $l$ type of neutrino to oscillate into a $k$ type of neutrino in vacuum is given by

$$
\begin{aligned}
P(l \rightarrow k)= & \delta_{l k}-4 \sum_{i<j} \operatorname{Re}\left(V_{k i}^{*} V_{l i} V_{l j}^{*} V_{k j}\right) \sin ^{2}\left(\frac{1.27 \Delta m_{i j}^{2}}{E} L\right) \\
& -2 \sum_{i<j} \operatorname{Im}\left(V_{k i}^{*} V_{l i} V_{l j} V_{k j}^{*}\right) \sin \left(\frac{2.54 \Delta m_{i j}^{2}}{E} L\right),
\end{aligned}
$$

where $\Delta m_{i j}^{2}$ is in $\mathrm{eV}^{2}, E$ in $\mathrm{GeV}$, and $L$ in $\mathrm{km}$. The first two terms are $C P$ conserving and symmetric in $l$ and $k$, while the last term is $C P$ violating and antisymmetric in $l$ and $k$. For antineutrino oscillation the last term will change sign.

In our new parametrization, to order $\epsilon^{2}$ we find the following oscillation probabilities:

$$
\begin{aligned}
P\left(\nu_{e} \rightarrow \nu_{e}\right)= & 1-4 \epsilon^{2}\left[\sin ^{2}\left(\frac{1.27 \Delta m_{12}^{2}}{E} L\right)\right. \\
& \left.+\sin ^{2}\left(1.27 \frac{\Delta m_{13}^{2}}{E} L\right)\right], \\
P\left(\nu_{\mu} \rightarrow \nu_{\mu}\right)= & P\left(\nu_{\tau} \rightarrow \nu_{\tau}\right)
\end{aligned}
$$




$$
\begin{aligned}
& =1-2 \epsilon^{2}\left[\sin ^{2}\left(\frac{1.27 \Delta m_{12}^{2}}{E} L\right)\right. \\
& \left.+\sin ^{2}\left(\frac{1.27 \Delta m_{13}^{2}}{E} L\right)\right] \\
& -\left(1-2 \epsilon^{2}\right) \sin ^{2}\left(\frac{1.27 \Delta m_{23}^{2}}{E} L\right) \text {, } \\
& P\left(\nu_{e} \rightarrow \nu_{\mu}\right)=P\left(\nu_{\tau} \rightarrow \nu_{e}\right)=2 \epsilon^{2}\left[\sin ^{2}\left(\frac{1.27 \Delta m_{12}^{2}}{E} L\right)\right. \\
& \left.+\sin ^{2}\left(\frac{1.27 \Delta m_{13}^{2}}{E} L\right)\right]+P^{-}, \\
& P\left(\nu_{\mu} \rightarrow \nu_{\tau}\right)=\left(1-2 \epsilon^{2}\right) \sin ^{2}\left(\frac{1.27 \Delta m_{23}^{2}}{E} L\right)+P^{-} \text {, } \\
& P^{-}=-4 \epsilon^{2} \sin \left(\frac{1.27 \Delta m_{12}^{2}}{E} L\right) \sin \left(\frac{1.27 \Delta m_{13}^{2}}{E} L\right) \\
& \times \sin \left(\frac{1.27 \Delta m_{23}^{2}}{E} L\right) \text {. }
\end{aligned}
$$

For the large $\nu_{\mu} \rightarrow \nu_{\tau}$ oscillation, further tests can be carried out to check the oscillation mechanism at the long base line neutrino experiments [9] at $\mathrm{K} 2 \mathrm{~K}$, Minos, and Opera in a controlled fashion. In these experiments, it may also be possible to carry out some tests for the predictions of the ansatz proposed here. In both cases (a) and (b), it is feasible to have the right combination of $L / E$ such that $1.27 \Delta m_{12}^{2} L / E$ or
$1.27 \Delta m_{13}^{2} L / E$ are of order unity so that oscillations between $\nu_{e}$ and $\nu_{\mu}$, and $\nu_{e}$ and $\nu_{\tau}$ can be observed. The long base line neutrino oscillation experiments $\mathrm{K} 2 \mathrm{~K}$, Minos, and Opera have $L$ around 250,730 , and $730 \mathrm{~km}$ with $E$ around $\sim 1.4$, $\sim 3,7,15$, and $\sim 17 \mathrm{GeV}$, respectively. It is possible using $E_{\nu_{\mu}}=3 \mathrm{GeV}$ from Minos to have $1.27 \Delta m_{12}^{2} L / E$ or $1.27 \Delta m_{13}^{2} L / E$ as large as $\pi / 2$. The maximal probability at the far end of the long base line detector for the appearance of $\nu_{e}$ can be as large as $2 \epsilon^{2}=(0.25-2.5) \times 10^{-3}$ from $\nu_{\mu}$ $\rightarrow \nu_{e}$ oscillation. The appearing probability of $\nu_{\mu}$ can be in the range $(0.25-2.5) \times 10^{-3}$ from $\nu_{e} \rightarrow \nu_{\mu}$ oscillation. Future experimental data will provide important information about the mixing matrix. Unfortunately, cases (a) and (b), to the leading order, give identical predictions for the abovementioned experiments and cannot be distinguished.

Another interesting prediction of the new mixing matrix parametrization is $C P$ violation in neutrino oscillations. Tests of $C P$ violation in neutrino oscillations may be feasible at neutrino factories [10,11]. Proposed neutrino factories have a high intensity muon storage ring with long sections along which the muons decay, $\mu^{-} \rightarrow \nu_{\mu} e^{-\bar{\nu}_{e}}$, to deliver high intensity $\nu_{\mu}$ and $\bar{\nu}_{e}$ neutrino beams. With these neutrino beams one can carry out disappearing and appearing neutrino experiments to test different neutrino mixing scenarios. Defining $C P$ violating asymmetry,

$$
A\left(\nu_{l} \rightarrow \nu_{k}\right)=\frac{P\left(\nu_{l} \rightarrow \nu_{k}\right)-P\left(\bar{\nu}_{l} \rightarrow \bar{\nu}_{k}\right)}{P\left(\nu_{l} \rightarrow \nu_{k}\right)+P\left(\bar{\nu}_{l} \rightarrow \bar{\nu}_{k}\right)}
$$

we have

$$
A\left(\nu_{e} \rightarrow \nu_{\mu}\right)=A\left(\nu_{\tau} \rightarrow \nu_{e}\right)=-A\left(\nu_{\mu} \rightarrow \nu_{e}\right)=-A\left(\nu_{e} \rightarrow \nu_{\tau}\right)=-2 \frac{\sin \left(\frac{1.27 \Delta m_{12}^{2}}{E} L\right) \sin \left(\frac{1.27 \Delta m_{13}^{2}}{E} L\right) \sin \left(\frac{1.27 \Delta m_{23}^{2}}{E} L\right)}{\sin ^{2}\left(\frac{1.27 \Delta m_{12}^{2}}{E} L\right)+\sin ^{2}\left(\frac{1.27 \Delta m_{13}^{2}}{E} L\right)} .
$$

In the above we have given the modes that may have large $C P$ violation. In principle, $A\left(\nu_{i} \rightarrow \nu_{i}\right), A\left(\nu_{\mu} \rightarrow \nu_{\tau}\right)$, and $A\left(\nu_{\tau} \rightarrow \nu_{\mu}\right)$ can have nonzero values. However, there is an additional suppression factor of order $\epsilon^{2}$ for $C P$ violation in these modes and they would be difficult to measure.

It is interesting to note that in the above expression, the mixing parameter $\epsilon$ has canceled out, and also that all the above modes have $C P$ violation of the same magnitude. Therefore, uncertainties associated with the mixing parameters do not enter and allow clear interpretations. These unique features can provide good tests for the new parametrization studied here.

Let us now study what value $A\left(\nu_{\mu} \rightarrow \nu_{e}\right)$ can have. One can tune $L / E$ to maximize the detection probability. Tuning
$1.27 \Delta m_{21}^{2} L / E=\pi / 2$ for case (a) and substituting $1.27 \Delta m_{31}^{2} L / E=\left(\Delta m_{31}^{2} / \Delta m_{21}^{2}\right) \pi / 2$, we have

$$
\begin{aligned}
A\left(\nu_{e} \rightarrow \nu_{\mu}\right) & =-2 \sin \left(\frac{\Delta m_{31}^{2}}{\Delta m_{21}^{2}} \frac{\pi}{2}\right) \approx-2 \frac{\Delta m_{31}^{2}}{\Delta m_{21}^{2}} \frac{\pi}{2} \\
& =-(0.2-2) \times 10^{-2} .
\end{aligned}
$$

In case (b), one would get the same magnitude, but opposite sign. This fact can be used to distinguish cases (a) and (b). To have a $3 \sigma$ detection of $C P$ asymmetry, one would need $\left(10^{10}-10^{8}\right)$ neutrino oscillation events.

When neutrino beams travel through the earth to reach the other side to be detected, due to the fact that the earth is 
composed of matter and not antimatter, the MSW effect for the neutrino and antineutrino beams is different. This results in additional asymmetry. To have some idea about the additional asymmetry due to matter effects, we consider case (b) using $m_{1}=0, m_{2}=2.5 \times 10^{-3} \mathrm{eV}, m_{3}=6 \times 10^{-2} \mathrm{eV}$, and $\epsilon^{2}=5 \times 10^{-4}$ as the input vacuum values for the neutrino masses and mixing for illustration. The matter effect is proportional to the electron density $N_{e}$ in the earth. The parameter that enters in the calculation is $A=2 \sqrt{2} G_{F} N_{e} E_{\nu}$. For neutrino oscillation, $A$ takes the positive sign and for antineutrino oscillation it takes the minus sign. For the typical neutrino energy of the neutrino factories and the electron density in the earth, $A$ is in the range of $10^{-7}-10^{-2} \mathrm{eV}^{2}$ [12]. We find that in vacuum for the above values of parameters, the $C P$ asymmetry is predicted to be 0.0055 . But with a nonzero $A$, the result can be very different. For example, for $A=10^{-3} \mathrm{eV}^{2}$, the asymmetry would be 0.035 . One should note that the asymmetry caused by the matter effect does not imply $C P$ violation in the mixing matrix. To pin down the effect due to $C P$ violation, one needs to have a good understanding of the profile of the earth to eliminate the asymmetry due to the MSW effect on neutrino oscillations. This is very difficult. However, we would like to point out that $A\left(\nu_{l} \rightarrow \nu_{k}\right)=\left[P\left(\nu_{l} \rightarrow \nu_{k}\right)-P\left(\bar{\nu}_{l} \rightarrow \bar{\nu}_{k}\right)\right] /\left[P\left(\nu_{l} \rightarrow \nu_{k}\right)\right.$ $\left.+P\left(\bar{\nu}_{l} \rightarrow \bar{\nu}_{k}\right)\right]$ can still provide important information about the neutrino mass hierarchy. Because in case (a) and case (b) the signs for $A\left(\nu_{e} \rightarrow \nu_{\mu}\right)$ and $A\left(\nu_{e} \rightarrow \nu_{\tau}\right)$ are different, and matter effects do not change the signs of the asymmetries for $A$ in the range $10^{-7}-10^{-2} \mathrm{eV}^{2}$.
We have considered a new parametrization for neutrino mixing matrix $V$ using the ansatz $V=\cos \theta I+i \sin \theta U$ with the value $\theta=\pi / 4$ which gives equal weight to the diagonal $(I)$ and nondiagonal $(U)$ pieces in $V$. This value of $\theta$ is a natural consequence of the atmospheric neutrino data which requires maximal $\nu_{\mu} \rightarrow \nu_{\tau}$ oscillation. The matrix $U$ depends on only one small parameter $\epsilon \sim 10^{-2}$ which gives the SMA solution to the solar neutrino problem. It also predicts that $P\left(\nu_{e} \rightarrow \nu_{\mu}\right)=P\left(\nu_{\tau} \rightarrow \nu_{e}\right)$ to the leading order.

We find that to order $\epsilon^{2}$, the $C P$ asymmetry $A\left(\nu_{e} \rightarrow \nu_{l}\right)$ is independent of $\epsilon$. Moreover, we find that the sign of $A\left(\nu_{e}\right.$ $\left.\rightarrow \nu_{\mu}\right)$ and $A\left(\nu_{e} \rightarrow \nu_{\tau}\right)$ could be used to distinguish between the two possible mass hierarchies, $m_{\nu_{\mu}}>m_{\nu_{\tau}}>m_{\nu_{e}}$ and $m_{\nu_{\tau}}$ $>m_{\nu_{\mu}}>m_{\nu_{e}}$, permitted by the SMA solution to the solar neutrino problem and other oscillation data, even though matter effects may affect the magnitude of the asymmetry significantly. These asymmetries could be detected at neutrino factories.

It is remarkable that our mixing matrix $V$ which contains only two parameters $(\theta$ and $\epsilon$ ) can explain the atmospheric and solar neutrino data. $C P$ asymmetry measurements in neutrino oscillations could provide future tests of our parametrization.

This work was supported in part by National Science Council under Grant No. NSC 89-2112-M-002-058 and in part by the Ministry of Education Academic Excellence Project 89-N-FA01-1-4-3. V.G. would like to thank the Department of Physics, National Taiwan University for hospitality while this work was being completed.
[1] M. Kobayashi and T. Maskawa, Prog. Theor. Phys. 49, 652 (1973).

[2] Z. Maki, M. Nakagawa, and S. Sakata, Prog. Theor. Phys. 28, 247 (1962); V.N. Gribov and B.M. Pontecorvo, Phys. Lett. 28B, 493 (1969).

[3] V. Gupta, Int. J. Mod. Phys. A 16, 1645 (2001).

[4] SuperK Collaboration, S. Fukuda et al., Phys. Rev. Lett. 81, 1562 (1998); 85, 3999 (2000); 86, 5651 (2001).

[5] SNO Collaboration, Q. Ahmad et al., Phys. Rev. Lett. 87, 071301 (2001).

[6] LSND Collaboration, A. Aguilar et al., hep-ex/0104049, and references therein.

[7] For reviews see, S.F. King, hep-ph/0105261; J. Ellis, hep-ph/0105265.

[8] L. Wolfenstein, Phys. Rev. D 17, 2369 (1978); S. Mikheyev and A. Smirnov, Sov. J. Nucl. Phys. 42, 913 (1985).

[9] K2K Collaboration, M. Sakuda et al., KEK-report-97-254;
K2K Collaboration, S.H. Ahn et al., Phys. Lett. B 511, 178 (2001); MINOS Collaboration, NUMI-L-375 (1998), http://www-numi.fnal.gov:8876/; OPERA Collaboration, M. Guler et al., CERN-SPSC-2000-028, CERN-SPSC-P-318, LNGS-P52-00(2000).

[10] B. Autin et al., CERN-SPSC-98-30; V. Barger et al., Phys. Rev. D 62, 073002 (2000); Y. Itow et al., http://psux1.kek.jp/ 50GeV-PS/Science.html; A. Bueno, M. Campanelli, and A. Rubbia, Nucl. Phys. B589, 577 (2000).

[11] S. Geer, hep-ph/0008155; T. Ota and J. Sato, Phys. Rev. D 63, 093004 (2001); H. Fritzsch and Z.-Z. Xing, hep-ph/0103242; M.C. Gonzalez-Garcia et al., hep-ph/0105159; J.J. GomezCadenas, Nucl. Phys. B (Proc. Suppl.) 99, 304 (2001).

[12] A. Dziewonski, in The Encyclopedia of Solid Earth Geophysics, edited by D. James (Van Nostrand Reinhold, New York, 1989), p. 331. 